\title{
Des médecins en bonne santé: de leurs études à la retraite
}

\section{Christine Romann}

Dr med., membre du Comité central de la FMH, responsable du département Promotion de la santé et prévention

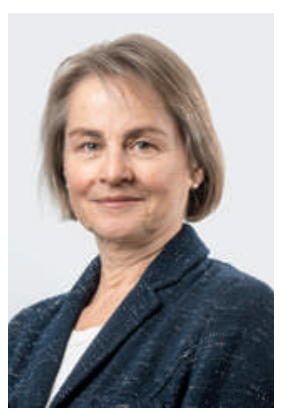

Malgré une charge de travail importante, les médecins sont majoritairement satisfaits de leur profession. Ils en apprécient la diversité et les exigences, ils ressentent la pertinence de leur activité et se réjouissent de l'estime qui leur est régulièrement accordée. Parce qu'ils peuvent concevoir et organiser leur travail en grande partie de manière autonome, ils sont très motivés et disposés à être performants.

Un emploi du temps chargé, des journées de travail interminables et la focalisation sur les besoins des patients comportent cependant aussi des risques: négliger, faute de temps, sa vie privée, son environne-

\section{Bien que les médecins en soient de facto les experts, ils veillent souvent trop peu à leur propre santé.}

ment social en dehors du cadre professionnel et le temps de récupération, fait le lit des stratégies de coping dysfonctionnelles et de l'épuisement progressif. Les changements des conditions de travail en cours depuis quelques années - perte d'autonomie, hausse des contrôles externes, tâches extraprofessionnelles, etc. restreignent la marge de manœuvre décisionnelle des médecins et leurs possibilités individuelles de s'organiser, au détriment de la satisfaction au travail.

La santé des médecins est pourtant un bien précieux, non seulement pour eux-mêmes, mais aussi pour les patients. En effet, des médecins surchargés et insatisfaits ont un impact sur la prise en charge médicale: en traitant leurs patients, ils courent plus fréquemment le risque de prendre une mauvaise décision, ils ne jouent plus le rôle d'exemple et occasionnent également des coûts liés à des arrêts maladie. Prendre soin de leur santé n'est dès lors pas uniquement du ressort des médecins eux-mêmes, mais relève aussi de la responsabilité de ceux qui ont la charge de leurs conditions de travail. C'est notamment aussi un bon investissement dans la qualité de la prévoyance santé.

Bien que les médecins soient, de facto, les experts de la santé, la manière de gérer la leur peut laisser à désirer: nombreux sont ceux qui manifestent une résistance à chercher une aide adéquate et à se faire soigner, et seule une minorité d'entre eux a un propre médecin de famille. Il n'est pas rare qu'un docteur se prescrive luimême ses propres médicaments - pas uniquement pour traiter des maux bénins - courant ainsi le risque de sous-estimer sa maladie, voire même de sombrer dans la dépendance.

En sa qualité d'association faîtière du corps médical, la FMH approfondira ce sujet lors du symposium «Des médecins en bonne santé: de leurs études à la retraite» qui se tiendra le 7 avril prochain à Berne. Au cœur de la rencontre se posera la question de savoir comment les médecins peuvent mieux veiller à leur propre santé pour résister aux multiples défis de leur profession sans aller jusqu'à l'épuisement. Il s'agira de les sensibiliser à leur propre santé mais aussi de rendre les employeurs comme les hôpitaux attentifs à ces questions. Le programme prévoit des exposés et des ateliers passionnants: la Dresse Andrée Rochfort, préposée à la qualité des généralistes irlandais et membre du comité du WONCA, nous parlera des expériences en Irlande et en Angleterre. Le Prof. Norbert Semmer, psychologue du travail, éclairera la question dans un contexte plus large et montrera les interférences entre travail et santé, notamment aussi dans les hôpitaux. Plusieurs ateliers sur les différentes phases de la carrière professionnelle d'un médecin, comme «survive studies combien de chemins mènent à Rome?» ou «Time-out

\section{Le symposium de la FMH du 7 avril 2016} entend sensibiliser les médecins, les hôpitaux et les formateurs à la santé des médecins.

contre Burnout», inviteront ensuite à la discussion avec experts et confrères sur les expériences passées et les solutions possibles.

Les conclusions de ce symposium jetteront les bases sur lesquelles se poursuivra l'engagement de la FMH sur ce terrain - apportez votre pierre à l'édifice en participant au symposium. Pour en savoir davantage, rendez-vous sur www.fmh.ch $\rightarrow$ Politique \& thèmes $\rightarrow$ Promotion de la santé et prévention $\rightarrow$ Symposium Médecins en bonne santé. 